\title{
Tumours with cancer stem cells: A PDE model
}

\author{
A. Fasano ${ }^{a, b, c}$, A. Mancini ${ }^{a, *}$, M. Primicerio ${ }^{a}$ \\ a Dipartimento di Matematica e Informatica U.Dini, Firenze, Italy \\ ${ }^{\mathrm{b}}$ IASI-CNR, Roma, Italy \\ ${ }^{c} R E D$ Department FIAB, Firenze, Italy
}

\section{A R T I C L E I N F O}

\section{Article history}

Received 7 September 2015

Revised 4 December 2015

Accepted 14 December 2015

Available online 21 December 2015

\section{Keywords:}

Stem cells

Cancer modelling

Tumour paradox

Reaction-diffusion systems

\begin{abstract}
A B S T R A C T
The role of cancer stem cells (CSC) in tumour growth has received increasing attention in the recent literature. Here we stem from an integro-differential system describing the evolution of a population of CSC and of ordinary (non-stem) tumour cells formulated and studied in a previous paper, and we investigate an approximation in which the system reduces to a pair of nonlinear coupled parabolic equation. We prove that the new system is well posed and we examine some general properties. Numerical simulations show more on the qualitative behaviour of the solutions, concerning in particular the so-called tumour paradox, according to which an increase of the mortality rate of ordinary (non-stem) tumour cells results asymptotically in a faster growth.
\end{abstract}

(c) 2015 Elsevier Inc. All rights reserved.

\section{Introduction}

In a recent paper [2] we considered a mathematical model (in the wake of $[8,10])$ that describes the growth of a tumour characterized by the presence of cancer stem cells (CSC). These cells, that have been identified in many cancers (see $[1,4,5,7,11,12]$ ), are practically immortal and are pluripotent, in the sense that after mitosis they can generate new CSC (a relatively rare event) or non-stem cancer cells (CC). ${ }^{1}$ On the contrary, CC can only generate $\mathrm{CC}$.

The model mentioned above consists in a system of integrodifferential equations that describe the evolution of the volume fractions of CSC and of CC, denoted by $u(x, t)$ and $v(x, t)$ respectively.

The system has the following form:

$\frac{\partial u(x, t)}{\partial t}=D \Delta u(x, t)+\delta \gamma \int_{\Omega} k(x, y, p(x, t)) u(y, t) d y, x \in \Omega, t>0$,

\footnotetext{
* Corresponding author. Tel.: +390552751403.

E-mail addresses: fasano@math.unifi.it (A. Fasano), mancini@math.unifi.it (A. Mancini), primicerio@math.unifi.it (M. Primicerio).

1 As a matter of fact, CSC can generate several lineages of non-stem cells [14]. We group all of them in a single sub-population since our main focus is on the role of CSC in the growth of the tumour.
}

$$
\begin{aligned}
\frac{\partial v(x, t)}{\partial t}= & D \Delta v(x, t)+(1-\delta) \gamma \int_{\Omega} k(x, y, p(x, t)) u(y, t) d y \\
& +\rho \int_{\Omega} k(x, y, p(x, t)) v(y, t) d y-\alpha v(x, t), x \in \Omega, t>0,
\end{aligned}
$$

with initial conditions

$$
\begin{aligned}
& u(x, 0)=u_{0}(x), \quad v(x, 0)=v_{0}(x), x \in \Omega, \\
& \left(u_{0}, v_{0} \in[0,1], u_{0}+v_{0} \leq 1\right) . \\
& \quad \text { In }(1.1) \text { and }(1.2) \\
& p(x, t)=u(x, t)+v(x, t) \\
& \quad \text { and: }
\end{aligned}
$$

- $\gamma, \rho$ are positive constants representing the replication rates of CSC and CC, respectively,

- $\delta, 0<\delta<1$, represents the fraction of symmetrical mitosis for CSC, i.e. the mitosis producing two newborn CSC. In most cases $\delta \ll 1$,

- $k(x, y, p(x, t))$ is the probability density that a cell located at $y$ generates a cell located at $x$,

- $D \geq 0$ is the diffusivity of the cells (see Remark 2.2 below),

- $\Omega$ is the domain where the phenomenon takes place.

The dependence of $k$ on the total cell population at $x, p(x, t)$, accounts for a crowding effect. In other words, we assume that larger 
values of $p$ at a given point induce a lower probability that a cell is generated at that point.

In the sequel, we will make the following natural assumptions:

$k(x, y, p(x, t))=F(p(x, t)) K(x, y), x, y \in \Omega, t>0$,

and we will suppose that

$K(x, y) \geqq 0, K \in C(\Omega \times \Omega)$

$F(p)$ is Lipschitz continuous in $[0,1], F(0)=1, F(1)=0$,

$F(p)$ is non-increasing and strictly decreasing at $p=1$.

Whenever necessary we will give $F(p)$ constant extensions out of the "physical" domain $[0,1]$.

The aim of this paper is to approximate (1.1) and (1.2) by a system of two coupled PDEs of parabolic type, forming a system of reaction-diffusion equations. The system will be shown to be well-posed, some qualitative properties will be analysed and some numerical simulations will be provided.

The plan of the paper is the following: in Section 2 we will show how (1.1) and (1.2) can be approximated by a reactiondiffusion system by an argument similar to the one discussed in [3]; in Section 3 we will discuss the well-posedness of the problem; in Section 4 we will give some numerical examples showing that the system exhibits the "tumour paradox" (i.e. larger mortality of CC may correspond to faster growth of the tumour).

\section{The reaction-diffusion model}

From now on, we refer to a one-dimensional case and we take $\Omega \equiv(-\infty,+\infty)$.

Moreover, we assume that function $K$ in (1.5) depends on $x$ and $y$ just through the distance between the two points, i.e.

$K(x, y)=K(|x-y|), x, y \in R$.

It will be a natural choice to assume that $K(z)$ is a decreasing function of its argument, with "small" variance, of the order - say - of a few cell diameters. An obvious example is

$K(x, y)=\sigma^{-1} \pi^{-1 / 2} \exp \left[-(x-y)^{2} / \sigma^{2}\right]$.

At this point, we can approximate the integral on the r.h.s. of (1.1) as follows:

$$
\begin{aligned}
& \int_{-\infty}^{+\infty} F(p(x, t)) K(|x-y|) u(y, t) d y \\
& \quad=F(p(x, t)) \int_{-\infty}^{+\infty} K(|x-y|) \\
& \quad \times\left[u(x, t)+(y-x) u_{x}(x, t)+(y-x)^{2} u_{x x}(x, t) / 2+\text { h.o.t. }\right] d y,
\end{aligned}
$$

so that, to the leading order

$$
\begin{aligned}
& \int_{-\infty}^{+\infty} F(p(x, t)) K(|x-y|) u(y, t) d y \cong F(p(x, t))[A u(x, t) \\
& \left.\quad+B u_{x x}(x, t)\right]
\end{aligned}
$$

where $A$ is the integral of $K$ and $B$ is half of its second order moment, while the first order moment cancels, due to the symmetry of $K$.

Of course, in case (2.2) we would have

$A=1, B=\sigma^{2} / 4$.

The same procedure can be applied to the integral involving $v$. Thus, we obtain the following system of reaction-diffusion equations:

$u_{t}=D u_{x x}+\delta \gamma F(p)\left[A u+B u_{x x}\right], x \in R, t>0$,

$$
\begin{aligned}
v_{t}= & D v_{x x}+(1-\delta) \gamma F(p)\left[A u+B u_{x x}\right] \\
& +\rho F(p)\left[A v+B v_{x x}\right]-\alpha v, \quad x \in R, t>0 .
\end{aligned}
$$

These two equations are supplemented with initial conditions

$u(x, 0)=u_{0}(x) \in[0,1]$

$v(x, 0)=v_{0}(x) \in[0,1], u_{0}+v_{0} \leq 1, x \in R$.

Obvious conditions at $\mp \infty$ are $u=v=0$.

Remark 2.1. It is clear that the integral term on the r.h.s of (1.1) is non-negative. However, after the approximation (2.3), we cannot take for granted that the second term on the r.h.s. of (2.5) is nonnegative too; indeed, we do not have a priori information on the sign and size of $B u_{x x}$. Nevertheless, $A u+B u_{x x}$ will be non-negative for "reasonable" initial data and for sufficiently "small" $\sigma$.

Remark 2.2. There is a large debate in the literature on the relevance of diffusive terms in (1.1) and (1.2). We believe that, in any case, if diffusion is taken into account it should depend on "crowding" and thus on $p$, and vanishing in the limit $p=1$. In the spirit of our approach, the diffusive terms, which is anyway very small, can be included in the coefficient $B$.

For this reason we will put $D=0$ in (2.5) and (2.6) from now on.

\section{An existence proof}

We will prove an existence result, locally in time.

Theorem 3.1. Let $u_{0}, v_{0} \in C^{2+\alpha}$ and such that, for any $x \in R$

$p_{0}(x)=u_{0}(x)+v_{0}(x) \leq 1-M, M \in(0,1)$,

then problem (2.5)-(2.7) has a classical solution $(u, v)$ in a suitably small time interval $\left(0, T^{*}\right)$ which is such that

$u(x, t)+v(x, t)<1$ in $R \times\left[0, T^{*}\right)$.

Proof. We use a fixed point argument.

We define the set $(u, v) \in \Sigma$ as follows: take $u, v$ both in $H^{\alpha, \frac{\alpha}{2}}(R \times(0, T))^{2}$ for some $T>0$, and such that $u(x, 0)=u_{0}(x)$, $v(x, 0)=v_{0}(x)$ for any $x$ in $R$.

Moreover, assume

$u+v \leq 1-N, \quad 0<N<M, \quad\|u\|^{\alpha},\|v\|^{\alpha}<K$,

for some $K>0$ and not less than $\left\|u_{0}\right\|^{\alpha},\left\|v_{0}\right\|^{\alpha}$. (1.8))

Take $(u, v) \in \Sigma$ and solve the uniformly parabolic system (recall

$U_{t}=\delta \gamma F(u+v)\left[A U+B U_{x x}\right], x \in R, t>0$,

$V_{t}=(1-\delta) \gamma F(u+v)\left[A U+B U_{x x}\right]$

$+\rho F(u+v)\left[A V+B V_{x x}\right]-\alpha V, x \in R, t>0$.

$U(x, 0)=u_{0}(x), \quad V(x, 0)=v_{0}(x), \quad x \in R$.

Eq. (3.4) can be solved independently of (3.5), providing $U \in$ $C^{2+\alpha}$ for $x \in R, t>0$ (see Theorem 8.1, p. 495 of [9]). Moreover the norm $\|U\|^{\alpha}$ can be estimated only in terms of $\left\|u_{0}\right\|^{\alpha}$ and $N$ (see Theorem 1.1, p. 419 of [9]).

Taking the function $Y(t)=\left\|u_{0}\right\| \exp (\delta \gamma A t)$, we consider the difference

$\omega(x, t)=Y(t)-U(x, t)$

and we find

$\omega_{t}-\delta \gamma F(u+v)\left(A \omega+B \omega_{x x}\right)=\delta \gamma A[1-F(u+v)] Y>0$.

\footnotetext{
${ }^{2}$ Here and in the following we use the notation of [9].
} 
$\mathrm{U}, \mathrm{t}=0.2000 \mathrm{E}+02$

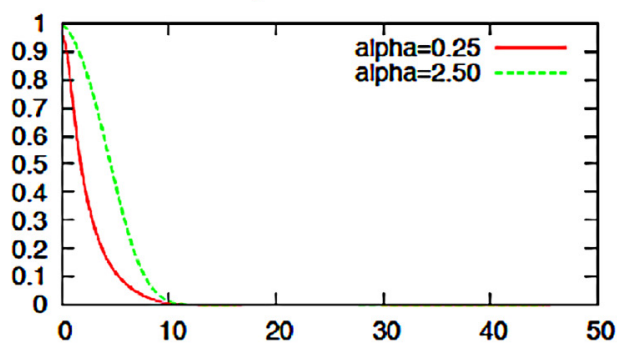

$\mathrm{U}+\mathrm{V}, \mathrm{t}=0.2000 \mathrm{E}+02$

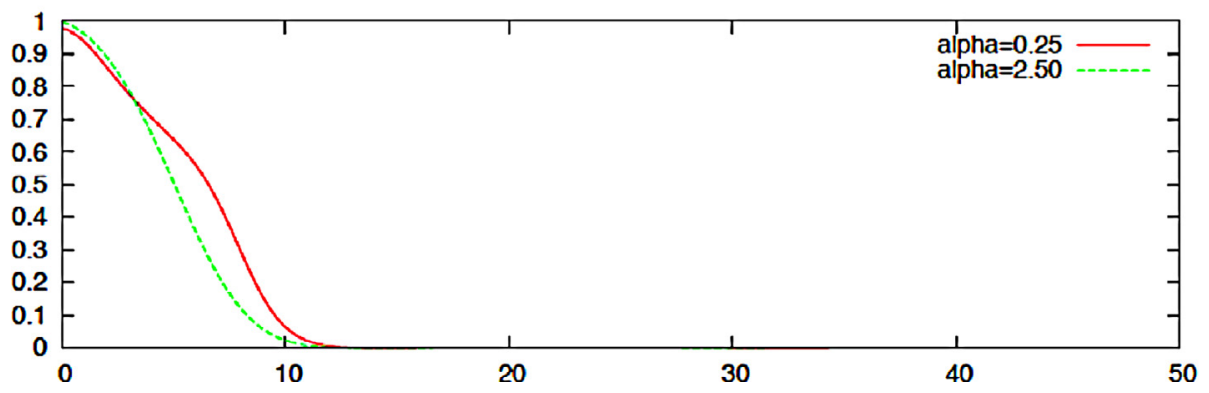

Fig. 1. $t=20$.

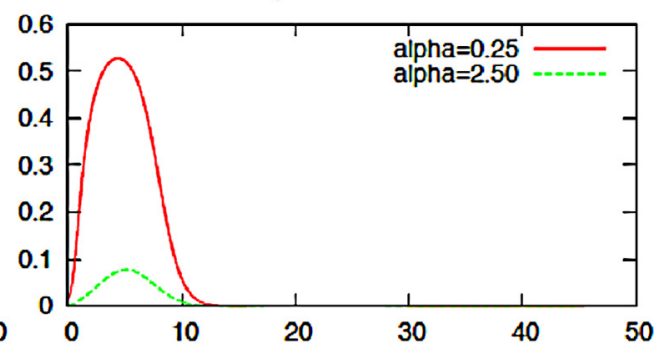

$V, t=0.3000 E+02$
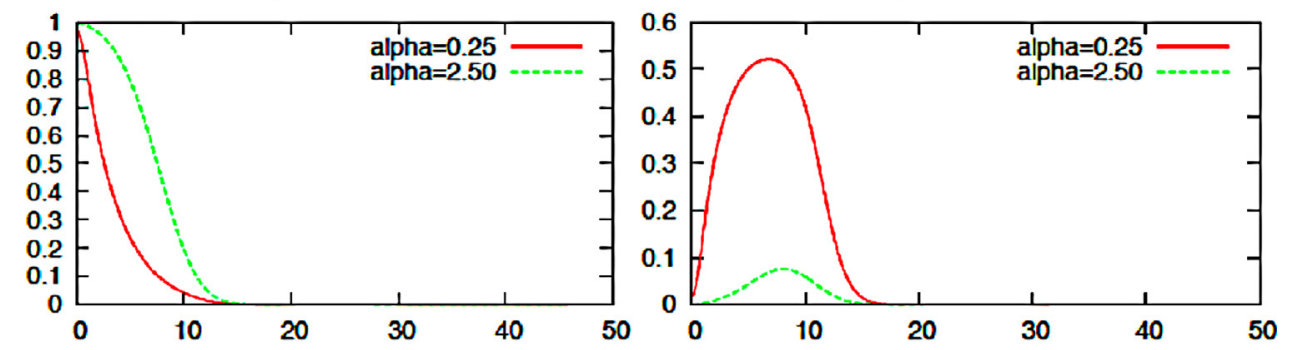

$U+V, t=0.3000 E+02$

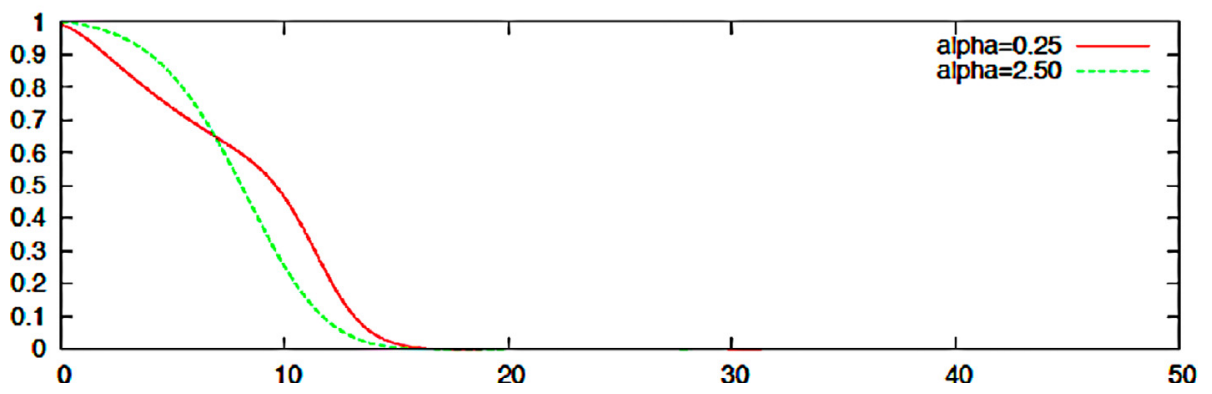

Fig. 2. $t=30$.

So we conclude that $\omega>0$ on the basis of Theorem 5, p. 39 of [6].

Thus $U \leq\left\|u_{0}\right\| \exp (\delta \gamma A t)$ and we can choose $T^{*}$ small enough to have

$U \leq\left\|u_{0}\right\|+\varepsilon_{1}$ in $R \times\left(0, T^{*}\right)$

for some positive $\varepsilon_{1}$.

Moreover, the norm $\|U\|^{2+\alpha}$ is estimated (see Theorem 8.1, p. 495 of [9]) in terms of $N, K$ and $\left\|u_{0}\right\|^{2+\alpha}$. This gives an estimate for the first term in the r.h.s. of (3.5). Therefore, we can possibly reduce $T^{*}$ to have

$V \leq\left\|v_{0}\right\|+\varepsilon_{2}$, in $R \times\left(0, T^{*}\right)$ for some positive $\varepsilon_{2}$, so that

$U+V \leq 1-N, \quad$ in $R \times\left(0, T^{*}\right)$.

It remains to get an estimate for $\left\|V^{\alpha}\right\|$. If we proceed as we did for $U$, the latter is going to depend on $\left\|U_{t}\right\|$. Similarly, $\|V\|^{2+\alpha}$ will depend on $\left\|U_{t}\right\|^{\alpha}$.

This means that it would be difficult to find a value of $K$ such that $\|V\|^{\alpha}<K$. Thus we proceed differently. Recalling again Theorem 1.1, p. 419 of [9] we realize that in order to get the desired estimate for $\|V\|^{\alpha}$ it is enough to start from an estimate of the norm $W^{(2,1)}$ of $U$. Now, Theorem 9.1, p. 341 of [9] provides the desired estimate, independent of $K$.

Next step to get the existence is to prove that the transformation $(u, v) \rightarrow(U, V)$ is a contraction in the norm of the uniform convergence, possibly in a smaller time interval. 
$\mathrm{U}, \mathrm{t}=0.4000 \mathrm{E}+02$

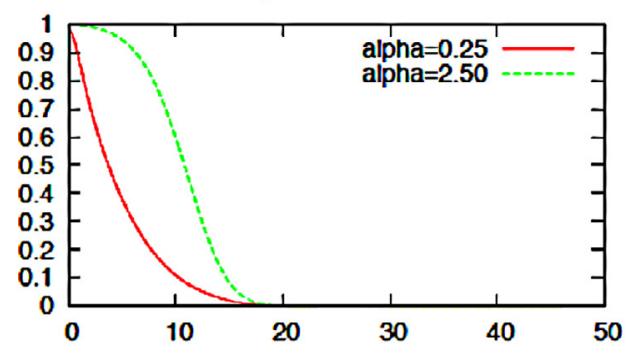

$\mathrm{U}+\mathrm{V}, \mathrm{t}=0.4000 \mathrm{E}+02$

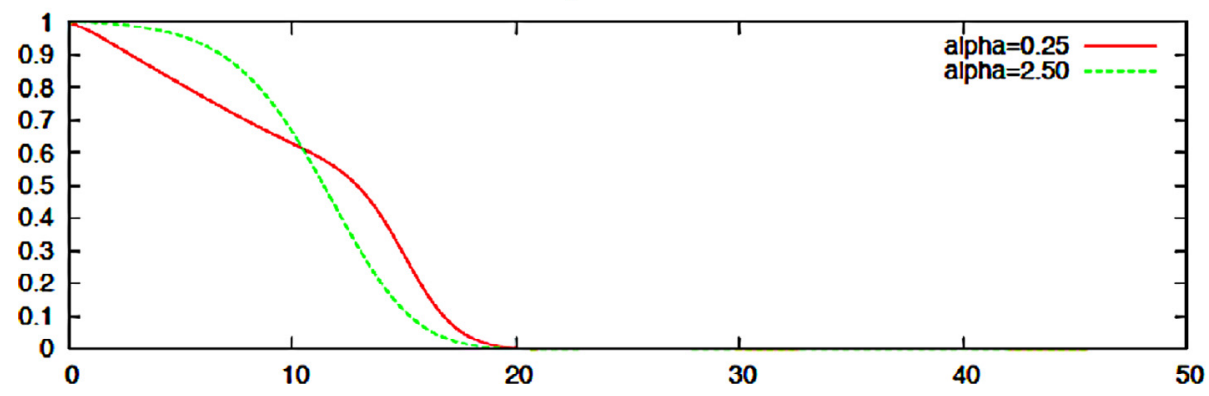

Fig. 3. $t=40$.

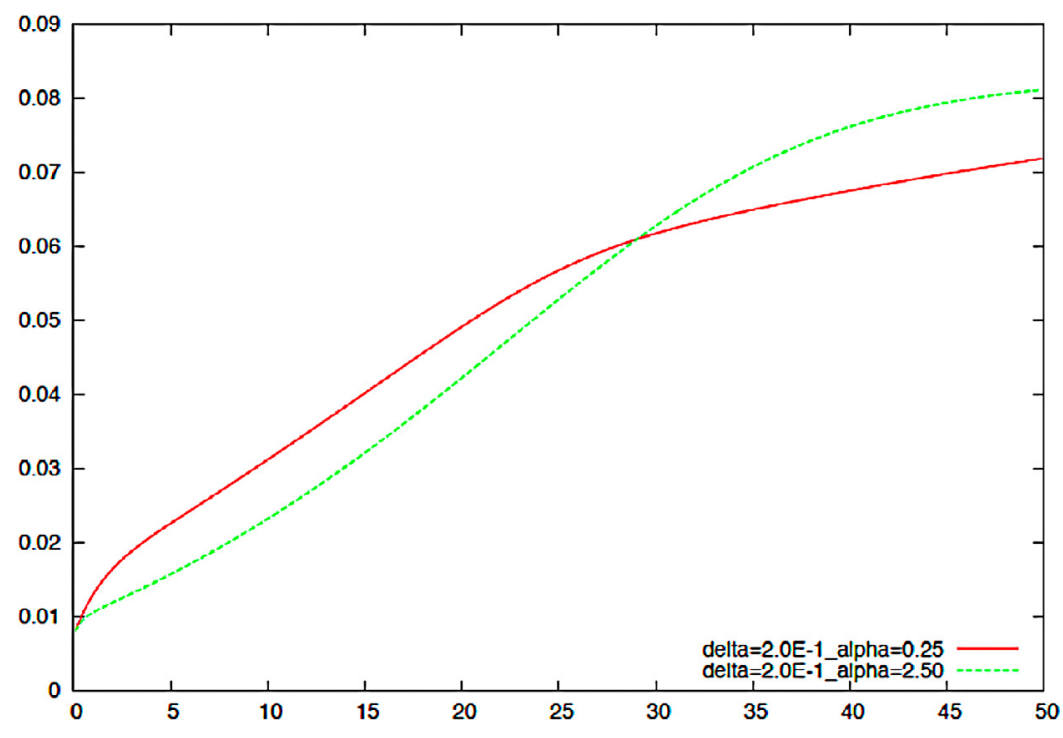

Fig. 4. Integral of $p=u+v$ as a function of time.

Indeed, take $\left(u_{1}, v_{1}\right)$ and $\left(u_{2}, v_{2}\right)$ in $\Sigma$ and consider the corresponding problems (3.4)-(3.6). Because of their linearity in $U$, $V$, it is easy to see that

$\left\|U_{1}-U_{2}\right\|_{0}+\left\|V_{1}-V_{2}\right\|_{0} \leq C T\left[|| u_{1}-u_{2}\left\|_{0}+\right\| v_{1}-v_{2} \|_{0}\right]$,

where $\|.\|_{0}$ is the uniform norm in $R \times(0, T)$ and the constant $C$ depends on the Lipschitz constant of $F$ and on the $C^{2+\alpha}$ norms of $U_{i}$ and $V_{i}$ that were already estimated in terms of $K$ and $N$. This means that, after a possible further reduction of $T^{*}$, the proof is concluded.

Remark 3.1. As a matter of fact the existence result just proved refers to a problem that is more general than the physical problem. Indeed, we did not use the assumptions on the positivity of the initial data. As a consequence, we did not get the positivity of $U$ and $V$. We can observe that the positivity of $U$ is an immediate consequence if $u_{0} \geq 0$ (see e.g. Theorem 5, p. 39 of [6]); but even for positive $v_{0}$ the positivity of $V$ is not granted unless we have that the second term on the r.h.s. of (2.3) is non-negative. As we noted in Remark 2.1 this is in fact true for reasonable initial data and in any case in a suitable time interval if $A u_{0}+B u_{0}^{\prime \prime} \geq \lambda>0$.

Remark 3.2. If we suppose that the solution we have found is such that $u_{t} \geq 0$ (thus implying $v>0$ ), then we can prove that it exists for all times.

Indeed, the existence proof can be iterated unless if $u+v$ reaches the value 1 . If this happens (for the first time) at some $\left(x^{*}, t^{*}\right)$, then $u_{t}\left(x^{*}, t^{*}\right)+v_{t}\left(x^{*}, t^{*}\right) \geq 0$. However, since $u_{t}\left(x^{*}, t^{*}\right)=0$, then $v_{t}$ has to be non-negative requiring $v\left(x^{*}, t^{*}\right)=0$.

However, on the other hand, we would also have

$u_{t} \leq \delta \gamma F(u)\left(A u+B u_{x x}\right), x \in R, t \in\left(0, t^{*}\right)$

and hence

$u(x, t) \leq Z(t) x \in R, \quad t \in\left(0, t^{*}\right)$, 


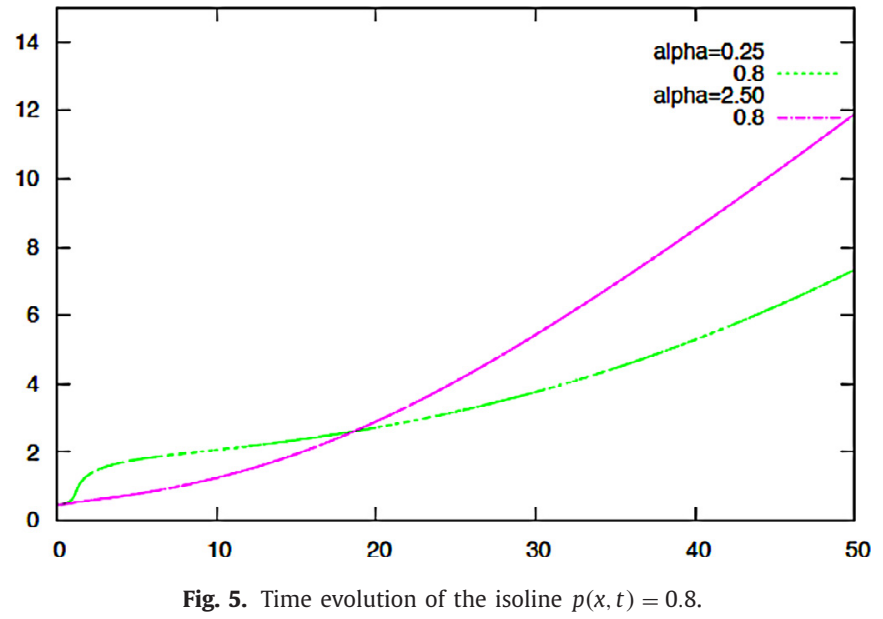

where $Z(t)$ is the solution of the ODE

$d Z / d t=A \delta \gamma F(Z) Z \quad t \in\left(0, t^{*}\right), Z(0)=\left\|u_{0}\right\|$,

and $Z(t)<1$ for any finite time, because of the Lipschitz continuity of $F$.

\section{Numerical examples}

We took $K$ as in (2.2), with different values of the widths $\sigma_{u}$ and $\sigma_{v}$ of the Gaussian curves. We calculated the solutions of (2.5)-(2.7) with different values of $\alpha$ (the mortality of CC), to see whether they show the tumour paradox.

For numerical purposes, we normalized space coordinate w.r.t. $\sigma_{u}$ and we calculated the solutions in the interval $(-50,50)$ that is much larger of the region occupied by the cells. We imposed noflux conditions on $-50,+50$. Initial conditions (in the space normalized variable) are

$u(x, 0)=\exp \left(-x^{2}\right), v(x, 0)=0, x \in(-50,50)$.

For symmetry reasons we can calculate the solution in $(0,50)$ with zero flux at the boundaries.

For all the numerical simulations we based our code on the fortran library BACOLR [13] due to its adaptive error control capabilities that resulted in a flexible and robust test bed.

Here we display some results obtained with the following values of the constants:

$\gamma=1, \quad \rho=1, \quad \delta=0.2, \quad \sigma_{u}=0.5, \quad \sigma_{v}=0.1$

and we considered two values for $\alpha, \alpha=0.25, \alpha=2.5$.

Figs. 1-3 show $u, v$ and $p=u+v$, as function of the (normalized) space coordinate, at non-dimensional times $t=20, t=30$, $t=40$, respectively.

Fig. 4 displays the integral of $p=u+v$ as a function of time. Finally, Fig. 5 shows the line where $p(x, t)=0.8$ in the $(x, t)$ plane. The simulation clearly exhibits the appearance of the tumour paradox.

\section{Conclusions}

We have described the evolution of a tumour where two different malignant populations are present: ordinary, non-stem cancer cells (CC) and cancer stem-cells (CSC). Starting from the integraldifferential formulation given in [2], a system of reaction-diffusion equations was obtained and its well-posedness was proved.

Numerical simulations were performed in order to emphasize the occurrence of the so called tumour paradox, a phenomenon characterized by an accelerated spread of cancer in presence of a higher CC mortality rate (e.g. drug-induced).

A first evidence of the paradox is displayed in Figs. 1-3, where the evolution of two tumours with different CC mortality rates (case (A): $\alpha=0.25$; case (B) $\alpha=0.5$ ) is compared.

Fig. 1 shows that at time $t=20$ the total population $p$ for case (A) is still everywhere larger than the one for case (B). The paradox is evident in Figs. 2 and 3 showing that an interval where $p_{B}>p_{A}$ appears and spreads.

The plots of $u$ and $v t=20,30$, and 40 show that a higher death rate for CC leaves more space for the invasion of CSC.

Finally, a very clear illustration of the paradox occurrence is provided by Figs. 4 and 5 . The former shows that the total tumoural mass in case (B) eventually supercedes the one corresponding to lower mortality rate. Fig. 5 shows that the line $p=0.8$ travel faster in case (B) than in case (A), meaning that the tumour expansion is paradoxically higher in case of higher CC mortality rate.

\section{Acknowledgements}

We thank the referees for their helpful comments and suggestions. The paper was partially supported by GNFM of INDAM.

\section{References}

[1] E. Beretta, V. Capasso, N. Morozova, Mathematical modelling of cancer stem cells population behavior, Math. Model. Nat. Phenom. 7 (1) (2012) 279-305, doi: $10.1051 / \mathrm{mmnp} / 20127113$.

[2] I. Borsi, A. Fasano, M. Primicerio, T. Hillen. A non-local model for cancer stem cells and the tumor growth paradox. Math. Med. Biol. first publihsed online November 20, 2015 doi:10.1093/imammb/dqv037.

[3] V. Capasso, G. Serio, A generalization of the Kermack-McKendrick deterministic epidemic model, Math. Biosci. 42 (1-2) (1978) 43-61. http://dx.doi.org/10.1016/ 0025-5564(78)90006-8.

[4] T. Dittmar, K. Zanker, Role of Cancer Stem Cells in Cancer Biology and Therapy, CRC Press, Boca Raton, 2013.

[5] H. Enderling, R. Anderson, M. Chaplain, A. Beheshti, L. Hlatky, P. Hahnfeldt, Paradoxical dependencies of tumor dormancy and progression on basic cell kinetics, Cancer Res. 69 (22) (2009) 8814-8821, doi:10.1158/0008-5472. CAN-09-2115.

[6] A. Friedman, Partial Differential Equations of Parabolic Type, Prentice-Hall, 1964.

[7] R. Ganguly, I.K. Puri, Mathematical model for the cancer stem cell hypothesis, Cell Prolif. 39 (1) (2006) 3-14, doi:10.1111/j.1365-2184.2006.00369.x.

[8] T. Hillen, H. Enderling, P. Hahnfeldt, The tumor growth paradox and immune system-mediated selection for cancer stem cells, Bull. Math. Biol. 75 (1) (2013) 161-184, doi:10.1007/s11538-012-9798-x.

[9] O. Ladyzhenskaia, V.S. ., N. Ural'tseva, Linear and Quasi-linear Equations of Parabolic Type, American Mathematical Society, Translations of Mathematical Monographs, American Mathematical Society, 1988.

[10] L. Maddalena, Analysis of an integro-differential system modeling tumor growth, Appl. Math. Comput. 245 (C) (2015) 152-157, doi:10.1016/j.amc.2014. 07.081.

[11] R. Sole, C. Rodriguez-Caso, T. Deisboeck, J.Saldance, Cancer stem cells as the engine of unstable tumor progression, J. Theor. Biol. 253 (4) (2008) 629-637. http://dx.doi.org/10.1016/j.jtbi.2008.03.034.

[12] T. Stiehl, A. Marciniak-Czochra, Mathematical modeling of leukemogenesis and cancer stem cell dynamics, Math. Model. Nat. Phenom. 7 (01) (2012) 166-202.

[13] R. Wang, P. Keast, P. Muir, Algorithm 874: Bacolr: Spatial and temporal error control software for PDEs based on high-order adaptive collocation, ACM Trans. Math. Softw. 34 (3) (2008) 15:1-15:28, doi:10.1145/1356052.1356056.

[14] H. Youssefpour, X. Li, A. Lander, J. Lowengrub, Multispecies model of cell lineages and feedback control in solid tumors, J. Theor. Biol. 304 (2012) 39-59. http://dx.doi.org/10.1016/j.jtbi.2012.02.030. 